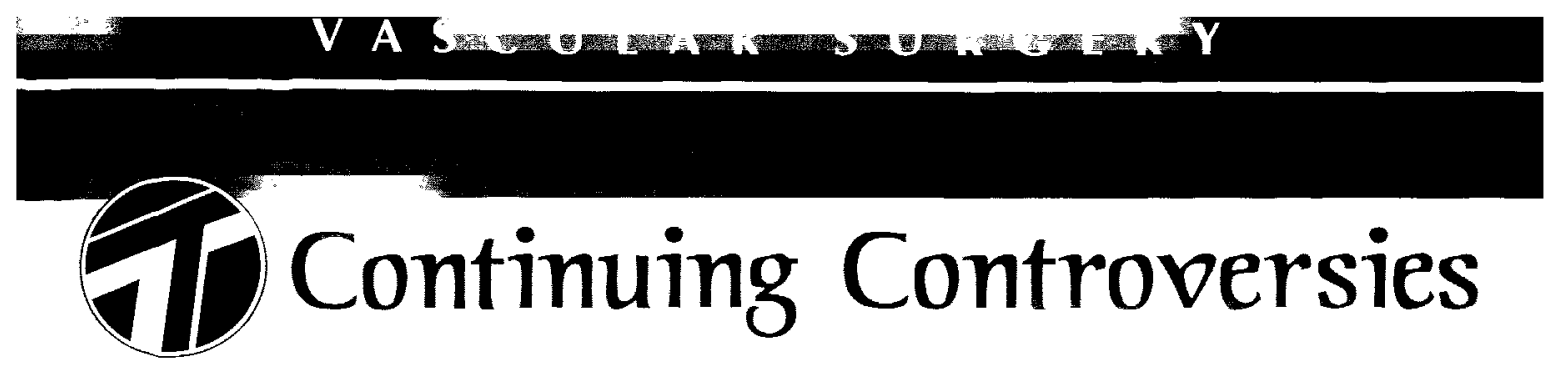

\title{
Surgery for Acute Stroke: Current Status
}

HUGH G. BEEBE, M.D., and BOONPRASIT KRITPRACHA, M.D.

ABSTRACT Although investigations regarding surgery for acute ischemic stroke span 4 decades, the topic remains controversial. This review considers emergency carotid surgery for acute stroke from four perspectives: an operation attempting to reverse an acute neurological deficit, an operation soon after acute stroke to prevent further neurologic deficit, reoperative surgery for acute neurological deficit early after carotid endarterectomy (CEA), and management of severe carotid stenosis after successful thrombolytic therapy for acute ischemic stroke. The available clinical data are absent or incomplete for each of these unusual aspects of carotid surgery. In the absence of a badly needed randomized trial, case selection is important and should be accompanied by a proven record of successful, low-morbidity, elective carotid surgery before undertaking more controversial indications. Delaying CEA for 4 to 6 weeks after stroke has repeatedly been shown to be unnecessary and to increase risk of interval new stroke. Finally, the impact of new thrombolytic treatment upon reoperative surgery for complications of CEA and for planning semielective reconstructive surgery needs to be considered.

Keywords Stroke, carotid endarterectomy, thrombosis

Perspectives in Vascular Surgery Vol. 10(1), pp. 85-94, 1999.

Surgery for acute ischemic stroke remains a controversial subject. Although investigations into this topic span 4 decades, the data we rely on for decision-

H.G.B., Director, Jobst Vascular Center, Toledo, OH; Clinical Professor of Surgery, University of Michigan Medical School, Ann Arbor, MI; B.K., Department of Vascular Surgerv, Songkla University, Hat-Yai, Songkla, Thailand, Visiting Research Scientist, University of Michigan, Ann Arbor, MI; Jobst Vascular Center, Toledo, OH. Copvright (C) 1999 by Thieme Medical Publishers, Inc., 333 Seventh Avenue, New York, NY 10001

Tel.: +1(212)760-0888.

All rights reserved. 
making guidance are unclear and often contradictory. In the early 1990s, panels convened by the Joint Council of the Society for Vascular Surgery, the North American Chapter of the International Society for Cardiovascular Surgery, and the American Heart Association could not develop firm conclusions regarding surgery for acute stroke based on the available data., ${ }^{1,2}$ Although recommendations were made for a randomized trial, to date no trials have been successfully implemented.

Indeed, prospects for a well-conceived randomized trial appear daunting. Mead et al attempted a randomized pilot study to ascertain whether urgent carotid surgery for acute stroke provided better outcomes than best medical care. ${ }^{3}$ During the 2 -year study period, only 16 patients were eligible for randomization from a total of 593 patients assessed. To replicate this trial with enough statistical power to detect a $20 \%$ improvement in outcome, a study would require 92 subjects. Assuming conditions similar to those of Mead's study, over 3400 patients would have to be assessed $(0.05 \alpha, 0.95 \beta$, unpaired $t$-test, difference of $0.20,3$ standard deviations). It is apparent that, although a randomized trial is justified by medical need and ethical standpoints, there are significant management and cost issues that would have to be resolved before a multicenter trial could be accomplished. Therefore, decisions concerning surgery for acute stroke must be based on available clinical data.

This review considers emergency carotid surgery for acute stroke from four perspectives: an operation attempting to reverse an acute neurological deficit, an operation soon after acute stroke to prevent further neurologic deficit, reoperative surgery for acute neurological deficit early after CEA, and management of severe carotid stenosis after successful thrombolytic therapy for acute ischemic stroke. Each of these unusual aspects of carotid surgery present unique challenges that require careful judgment. The available clinical data are absent or incomplete for all of them.

\section{SURGERY WITH THE INTENTION OF REVERSING NEUROLOGICAL DEFICIT}

Timing is a critical factor when considering surgery for the purpose of reversing or reducing a neurological deficit. Unfortunately, there are very little data that document the limits of critical timing between occlusion of the internal carotid artery (ICA) and attempts to restore blood flow that are successful for both hemodynamic and neurologic results. The notion that interruption of cerebral blood flow results in cellular death within minutes is simplistic and fails to recognize the clinical implications of the ischemic penumbra concept. A full discussion of this topic is beyond the intent of this article. There are experimental animal data, and clinical data consistent with them, to support the notion that a zone of cerebral tissue ischemia exists 
after acute arterial occlusion that may be reversed by timely intervention. The temporal limits of potential reversibility are not well defined in humans, not practically identifiable by any existing test methods, and probably have individual variability. An arbitrary selection of less than 4 hours from onset of neurological symptoms has been applied by some, ${ }^{4}$ but others have allowed longer intervals. ${ }^{5}$

One problem that pervades urgent intervention for stroke is the inability to know whether a deficit treated by carotid surgery within a few hours would have spontaneously cleared without surgery at all. Although it is true that most transient ischemic attack (TIA) symptoms clear within minutes to less than 2 hours, the literature continues to apply the criterion of 24 hours to define transient ischemia. Certainly, if carotid surgery is being contemplated specifically for the purpose of attempting to reverse an acute neurological deficit, reducing delay makes obvious theoretical sense. Early reports of successful interdiction of evolving stroke by emergency carotid surgery include the completely successful outcome in 26 patients reported in $1978 .^{\circ}$ Emergency carotid surgery was performed in that study, but only qualitative retrospective neurological assessment was provided.

The following criteria should be considered when determining eligibility for emergency CEA less than 24 hours after stroke: mild to moderate neurological deficit, cardiovascular stability during the period of observation, and ultrasound or angiography evidence of significant carotid stenosis or occlusion in the ipsilateral artery. Contraindications to surgery during this period include profound neurological deficit with or without loss of consciousness, cardiovascular instability, and computerized axial tomography (CT) scan evidence of major area of infarction or increased intracranial pressure or hemorrhage.

Investigators have reported varying degrees of success when attempting to reverse neurological defects via surgery. Meyer et al described 34 patients with acute onset of profound neurological deficits resulting from acute ICA occlusion. ${ }^{5}$ Because all of these patients were already hospitalized for investigation or preoperative preparations, most had surgery within hours of the cerebrovascular event. Overall, all but two ICAs were reopened, and a majority of patients improved. The death rate was $20.6 \%(7 / 34)$, however. It is important to note that 16 of 34 patients $(47 \%)$ with any neurological improvement were operated on in 6 hours or less, the majority in less than 4 hours. Of the seven deaths only two were due to cerebral hemorrhage, and both of these were operated upon within 6 hours. The specter of brain hemorrhage has cast a very dark shadow on emergency carotid surgery since the early reports of Wylie and Rob soon after carotid reconstructive surgery was described. ${ }^{7,8}$ However, so much improvement in hospital care, blood pressure control, and technical vascular surgery has occurred in the past 30 years that the subject can legitimately be kept open today. 
Such studies provide a registry of cases, not a clinical trial, and the results do not form a foundation for recommendation as a proven treatment for the following reasons:

1. Neurological deficit scoring is not used.

2. The length of time from onset of neurological deficit is poorly recorded.

3. The length of time postoperatively required for neurological improvement is virtually undescribed.

4. Medical therapy alone in stroke patients with mild/moderate neurological deficit results in improvement in a majority of patients within 2 weeks.

The grading and classification of acute neurological deficits are somewhat vague and poorly understood by many vascular surgeons. Part of the difficulty is the lack of observer continuity. In a typical case, stroke symptoms may be described variably by the patient, the family, emergency department exams, primary physicians, neurologists, and hospital nurses performing neurological assessments. The terms stroke in evolution, progressive stroke, stuttering stroke, fluctuating deficit, reversible ischemic neurologic deficit, acute stroke with rapid improvement, and crescendo TIA have all been used to indicate how unstable neurological conditions thought due to ischemia are described.

Although there is not full agreement in the existing literature, many experienced clinicians advise against considering emergency surgical therapy for any acute neurological deficit. Others suggest excluding patients with progressive neurological deficit as distinct from fluctuating ones with intervals of clearing. In the controversial and completely unsettled area of carotid surgery to relieve stroke, the surgeon contemplating emergency intervention should be cautious. The scrutiny of surgical results in carotid disease has been appropriately heightened in recent years by published results of low morbidity and mortality rates from many centers accompanied by guidelines from interdisciplinary national sources. Therefore, before embarking on aggressive emergency surgery, it would seem prudent to establish an experience with successful CEA for traditional, conservative indications.

\section{SURGERY TO PREVENT DEFECTS}

CEA is often delayed for 4 to 6 weeks after the onset of stroke. This approach is largely based on reports from the 1960s that had poor outcomes and high mortality for the operation in acute stroke patients. ${ }^{9}$ Reaction to cautionary early reports of experience with emergency surgery lead to adoption of a conservative approach with an arbitrary delay between onset of the stroke and subsequent carotid surgery. The interval of 6 weeks became a widespread 
standard but lacked a clinically validated basis. One of the early suggestions that arbitrary delay might be unnecessary in all patients and accompanied by significant risk came from Dosick et al, who observed that 4 of 19 patients (21\%) sustained a second denser episode during the 4- to 6-week waiting period. ${ }^{10} \mathrm{He}$ and his colleagues used CT scans of the brain to determine timing of CEA in stroke patients; 245 patients with acute stroke underwent brain CT 1 and 5 days after the initial event. Seventy percent (171 patients) were found to have negative CT, and $64 \%$ (110 patients) had appropriate carotid lesions for surgery demonstrated by angiography. All 110 patients underwent CEA within 14 days (mean 10 days) and only 1 postoperative stroke was observed. Meanwhile, among the 30\% (74 patients) with CT positive scans and a 4- to 6-week waiting period before CEA, 9.5\% (7 patients) suffered recurrence of cerebral infarction with 1 death. Ricotta et al had a similar encouraging result among 17 CT negative patients undergoing urgent CEA in less than 10 days after the onset of stroke. ${ }^{11}$

Sacco et al studied 1273 patients with cerebral infarction as part of the Stroke Data Bank of the National Institute of Neurological Disorders and Stroke (NINDS). ${ }^{12}$ The risk of reinfarction within 30 days after the first onset of stroke due to carotid stenosis or occlusion was 7.9\%. Among the 103 medically treated patients in the North American Symptomatic Carotid Endarterectomy Trial diagnosed with symptoms of stroke or severe stenosis, $4.9 \%$ had a recurrent ipsilateral stroke within 30 days after entry into the trial. Gasecki et al reviewed 100 CEA patients after they suffered nondisabling strokes. ${ }^{13}$ The patients were classified into 2 groups: 42 patients who underwent CEA within 30 days after stroke and 58 patients who underwent operation beyond 30 days after stroke. The morbidity and mortality were comparable in these two groups. The investigators concluded that delaying surgery in such cases may needlessly place patients at a risk for a recurrent stroke.

Piotrowski et al performed CEA at variable intervals in 129 patients after stroke. The investigators suggested that, using a plateau of neurological recovery, early CEA can be performed with no increase in morbidity and mortality. ${ }^{14}$ An arbitrary delay of 6 weeks was not required to prevent perioperative complications. Whittemore et al also had a good result of early CEA in patients with small, stable stroke and significant carotid stenosis $(>75 \%) .{ }^{15}$

In stable, nondisabling stroke with or without small infarction documented in brain CT, delaying 4 to 6 weeks will put patients at $4.9 \%$ to $21 \%$ risk of reinfarction and does not prevent perioperative neurological complications. Patients whose neurological deficit has cleared or become stable with a moderate deficit arising ipsilateral to carotid stenosis are at risk of further functional loss. Prompt operation should be considered in those with cardiovascular stability and absence of CT evidence of major infarction, hemorrhage, or intracranial pressure increase on an appropriately timed examination. 


\section{STROKE AFTER SUCCESSFUL CAROTID ENDARTERECTOMY}

Patients who sustain a new neurological deficit following CEA represent a special category in the management of acute stroke in many respects. First, their neurological status is both well known and subject to intensive observation. Most new neurological deficits after carotid surgery occur within 2 to 4 hours, and almost all deficits occur within 24 hours. Patients who have sustained a stroke followed by good functional recovery after CEA under general anesthesia often appear to briefly demonstrate their previous neurological deficit or parts of it during emergence from anesthesia. This finding resolves quickly in the recovery room but should be kept in mind. However, in general, the acute neurological deficit after CEA follows a period during which time the patient has been alert and neurologically stable in comparison to their preoperative status.

There are many causes of early postoperative stroke, and these have been usefully classified by Riles et al into five categories of 20 different mechanisms. ${ }^{16}$ Most, however, will be an acute neurological change within hours of straightforward CEA. Modern carotid surgery has achieved very low rates of early postoperative stroke, so their management is not a matter of routine. ${ }^{17-20}$ Thus, there is a tendency for individual evaluation to occur that may result in significant time spent obtaining external ultrasound (performed under difficult technical circumstances) or worse, arteriography. Although not everyone agrees, most recent reports have supported the strategy of immediate reoperation without diagnostic procedures other than bedside clinical evaluation by the operating surgeon.

Proponents of detailed CEA completion imaging by angiography or operative ultrasonography may doubt that a technical fault will be found that can be corrected by reoperation. However, this leaves unapproached the issue of platelet aggregates collecting on the fresh endarterectomy surface of an otherwise technically perfect operation.

\section{Technical Points in Carotid Surgery for Acute Stroke}

Detailed information about technical surgery is best left to an atlas format, but some aspects seem relevant to this discussion. The presence of an acute thrombosis or a partially occluding thrombus sometimes found during arteriography, together with early reoperation, all require the greatest sensitivity to disciplined, careful tissue handling during the procedure. A strict "notouch" technique with clamping of the ICA before any manipulation aids embolism protection. Often cerebral collaterals will provide enough arterial back pressure to "blow out" a thrombus from the unclamped, opened ICA. The use of balloon catheters for distal thrombus extraction is dangerous but may be attempted if kept below the level of the carotid sinus where iatrogenic trauma, even with a delicate touch, may produce arteriovenous fistula. 
The possibility of heparin antibody reaction should be considered in the post-CEA patient being returned to the operating room. Even if the preceding CEA was the patient's first cardiovascular operation, prior exposure to heparin may have occurred because of its ubiquitous quality in modern hospitals. Therefore, the reflex administration of heparin while returning the patient to the operating room may have its hazards. The use of low molecular weight dextran with a loading dose begs the question until the artery can be opened. The presence of the typical clumping of pinkish gray platelet aggregates in the endarterectomy site, together with absence of an identifiable technical fault that is correctable, makes the presumptive diagnosis of heparin antibody-induced platelet aggregation advisable until subsequent tests can be done. Vigorous local measures to clean the endarterectomy surface should include local irrigation with low molecular weight dextran as well.

The adjunctive use of thrombolytic drugs during carotid surgery has recently been reported by Comerota and Eze, whose patient sustained an acute hemiplegia initially detected upon awakening after CEA. ${ }^{21}$ Finding no technical flaw and a patent ICA, they infused 1 million units of urokinase over 1 hour to treat an occluded anterior communicating artery shown on operative arteriography. The resolution of the hemiplegia was immediate, although the patient sustained cerebral infarction in the posterior circulation demonstrated later.

\section{PATIENTS WITH ACUTE STROKE RESOLVED BY THROMBOLYTIC THERAPY}

Increasing reports of successful thrombolytic treatment for acute stroke are appearing, with successful recanalization of various cerebral blood supply vessels reported. ${ }^{22-24}$ The NINDS tissue plasminogen activator trial describes "large vessel" occlusive disease in 252 of 624 randomized patients followed for 3 months. ${ }^{25}$ Unfortunately, extracranial carotid disease is not specifically identified in this report. The report also does not inform whether any of these patients received anticoagulation treatment beyond the initial 24 hours when its use was apparently proscribed by the study protocol. The experience reported by del Zoppo et al describes $\mathbf{5 7 . 7 \%}$ resolution of acute occlusion in 26 patients treated with rpro-UK, although with a doubling of cerebral hemorrhage risk to $15.4 \%$ compared with placebo. ${ }^{24}$ They only recognized internal carotid occlusion in $8 \%$ of patients.

The advent of this approach to management of acute stroke seems certain to uncover a significant number of patients who will be found to have a severe carotid stenosis after the neurological deficit has been cleared. This presents a new category of patients for the vascular surgeon to consider. Given the increased risk of cerebral hemorrhage that has accompanied the beneficial results of these thrombolytic trials, and the likelihood that if 
carotid stenosis is present it will be very severe, will the risk of restoring normal blood pressure perfusion be increased? In the absence of data, it would seem that this special circumstance represents an area where conservatism is appropriate and a period of stabilization with or without anticoagulation might be prudent.

\section{CONCLUSION}

The message of the reported experience in recent years is that acute stroke is an area where carotid surgery has a contribution to make in resolving neurological deficit. In the absence of a badly needed randomized trial, case selection is important and should definitely be accompanied by a proven record of successful, low-morbidity, elective carotid surgery before undertaking more controversial indications.

Delaying CEA for arbitrary intervals of 4 to 6 weeks after stroke has repeatedly been shown to be unnecessary and to increase risk of interval new stroke.

The impact of new thrombolytic treatment upon reoperative surgery for complications of CEA and for planning semielective reconstructive surgery needs to be considered.

\section{REFERENCES}

1. Moore WS, Mohr JP, Najafi H, Robertson JT, Stoney RJ, Toole JF. Carotid endarterectomy: practice guidelines. Report of the Ad Hoc Committee to the Joint Council of the Society for Vascular Surgery and the North American Chapter of the International Society for Cardiovascular Surgery. J Vasc Surg 1992;15:469-479

2. Moore WS, Barnett HJ, Beebe HG, et al. Guidelines for carotid endarterectomy. A multidisciplinary consensus statement from the Ad Hoc Committee, American Heart Association. Circulation 1995;91:566-579

3. Mead GE, Murray H, Farrell A, O'Neill PA, McCollum CN. Pilot study of carotid surgery for acute stroke. Br J Surg 1997;84:990-992

4. Najafi H, Javid H, Dye WS, Hunter JA, Wideman FE, Julian OC. Emergency carotid thromboendarterectomy. Arch Surg 1971;103:610-614

5. Meyer FB, Sundt TM Jr, Piepgras DG, Sandok BA, Forbes G. Emergency carotid endarterectomy for patients with acute carotid occlusion and profound neurological deficits. Ann Surg 1986;203:82-89

6. Goldstone J, Moore WS. A new look at emergency carotid artery operations for the treatment of cerebrovascular insufficiency. Stroke 1978;9:599-602

7. Wylie EJ, Hein MF, Adams JE. Intracranial hemorrhage following surgical revascularization for treatment of acute strokes. J Neurosurg 1964;21:212-215

8. Rob CG. Operation for acute completed stroke due to thrombosis of the internal carotid artery. Surgery 1969;65:862-865

9. Beebe HG. Surgery for acute stroke. Sem Vasc Surg 1995;8:55-61

10. Dosick SM, Whalen RC, Gale SS, Brown OW. Carotid endarterectomy in the stroke patient: computerized axial tomography to determine timing. J Vasc Surg 1985; 2:214-219 
11. Ricotta JJ, Ouriel K, Green RM, DeWeese JA. Use of compterized cerebral tomography in selection of patients for elective and urgent carotid endarterectomy. Ann Surg 1985; 202:783-787

12. Sacco RL, Foulkes MA, Mohr JP, Wolf PA, Hier DB, Price TR. Determinants of early recurrence of cerebral infarction. The stroke data bank. Stroke 1989;20:983-989

13. Gasecki AP, Ferguson GG, Eliasziw M, Clagett GP, Fox AJ, Hachinski V, Barnett HJM. Early endarterectomy for severe carotid artery stenosis after a nondisabling stroke: results from the North American Symptomatic Carotid Endarterectomy Trial. J Vasc Surg $1994 ; 20: 288-295$

14. Piotrowski JJ, Bernhard VM, Rubin JR, McIntyre KE, Malone JM, Parent FN III, Hunter GC. Timing of carotid endarterectomy after acute stroke. J Vasc Surg 1990; $11: 45-52$

15. Whittemore AD, Ruby ST, Couch NP, Mannick JA. Early carotid endarterectomy in patients with small, fixed neurologic deficits. J Vasc Surg 1984;1:795-799

16. Riles TS, Imparato AM, Jacobowitz GR, Lamparello PJ, Giangola G, Adelman MA, Landis R. The cause of perioperative stroke after carotid endarterectomy. J Vase Surg 1994;19:206-216

17. Goldstein LB, Samsa GP, Matchar DB, Oddone EZ. Multicenter review of preoperative risk factors for endarterectomy for asymptomatic carotid artery stenosis. Stroke $1998 ; 29: 750-753$

18. Hertzer NR, O'Hara PJ, Mascha EJ, Krajewski LP, Sullivan TM, Beven EG. Early outcome assessment for 2228 consecutive carotid endarterectomy procedures: the Cleveland Clinic experience from 1989 to 1995 . J Vasc Surg 1997;26:1-10

19. Perler BA, Dardik A, Burleyson GP, Gordon TA, Williams GM. Influence of age and hospital volume on the results of carotid endarterectomy: a statewide analysis of 9918 cases. J Vasc Surg 1998;27:25-33

20. Maxwell JG, Rutledge R, Covington DL, Churchill MP, Clancy TV. A statewide, hospital-based analysis of frequency and outcomes in carotid endarterectomy. Am J Surg 1997; 174:655-660

21. Comerota AJ, Eze AR. Intraoperative high-dose regional urokinase infusion for cerebrovascular occlusion after carotid endarterectomy. J Vasc Surg 1996;24:1008-1016

22. Mori E, Tabuchi M, Yoshida T, Yamadori A. Intracarotid urokinase with thromboembolic occlusion of the middle cerebral artery. Stroke $1988 ; 19: 802-812$

23. Barr JD, Horowitz MB, Mathis JM, Sclabassi RJ, Yonas H. Intraoperative urokinase infusion for embolic stroke during carotid endarterectomy. Neurosurgery 1995;36:606-611

24. del Zoppo GJ, Higashida RT, Furlan AJ, Pessin MS, Rowley HA, Gent M. PROACT: a phase II randomized trial of recombinant pro-urokinase by direct arterial delivery in acute middle cerebral artery stroke. PROACT investigators. Prolyse in acute cerebral thromboembolism. Stroke 1998;29:4-11

25. The National Institute of Neurological Disorders and Stroke rt-PA Stroke Study Group. Tissue plasminogen activator for acute ischemic stroke. N Engl J Med 1995; 333:1581-1587 